\title{
Analysis of Raster Scanning Damage and Conditioning Experiments
}

\author{
M.D. Feit, and A.M. Rubenchik
}

This article was submitted to Boulder Damage Symposium XXXIV: Annual Symposium on Optical Material for High Power Lasers, Boulder, Colorado, September 16-18, 2002

\section{October 11, 2002}

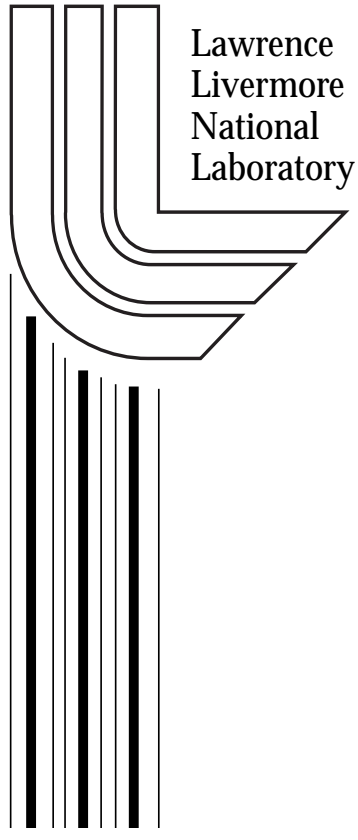




\section{DISCLAIMER}

This document was prepared as an account of work sponsored by an agency of the United States Government. Neither the United States Government nor the University of California nor any of their employees, makes any warranty, express or implied, or assumes any legal liability or responsibility for the accuracy, completeness, or usefulness of any information, apparatus, product, or process disclosed, or represents that its use would not infringe privately owned rights. Reference herein to any specific commercial product, process, or service by trade name, trademark, manufacturer, or otherwise, does not necessarily constitute or imply its endorsement, recommendation, or favoring by the United States Government or the University of California. The views and opinions of authors expressed herein do not necessarily state or reflect those of the United States Government or the University of California, and shall not be used for advertising or product endorsement purposes.

This work was performed under the auspices of the U. S. Department of Energy by the University of California, Lawrence Livermore National Laboratory under Contract No. W-7405-Eng-48.

This report has been reproduced

directly from the best available copy.

Available to DOE and DOE contractors from the

Office of Scientific and Technical Information

P.O. Box 62, Oak Ridge, TN 37831

Prices available from (423) 576-8401

http:/ /apollo.osti.gov/bridge/

Available to the public from the

National Technical Information Service

U.S. Department of Commerce

5285 Port Royal Rd.,

Springfield, VA 22161

http://www.ntis.gov/

OR

Lawrence Livermore National Laboratory

Technical Information Department's Digital Library

http://www.llnl.gov/tid/Library.html 


\title{
Analysis of raster scanning damage and conditioning experiments
}

\author{
M. D. Feit and A.M. Rubenchik* \\ University of California \\ Lawrence Livermore National Laboratory, \\ P.O. Box 808-L399, Livermore, CA. 94550
}

\begin{abstract}
The raster scan technique is used for large optics damage tests and laser conditioning. We show that the "effective area" concept enables the possibility to compare various scanning schemes and to use raster scan experiments for NIF optics damage prediction. It is shown that the hexagonal lattice of laser beam imprints yields optimal use of each shot for most of the typically used parameters. The effects of beam fluence fluctuations and pointing inaccuracies on experiments are evaluated. To analyze raster scan conditioning experiments, we introduce the concept of "effective dose", i.e. total dose averaged over a unit cell of the scan lattice. This allows various scanning schemes to be compared quantitatively.
\end{abstract}

\section{INTRODUCTION}

The high quality UV optics which will be used in high power laser systems such as NIF and LMJ are expected to have as little as one damaging defect per $100 \mathrm{~cm}^{2}$ at operating fluences [1]. To have reliable characterization of damage susceptibility of such optics, an area of a few hundreds $\mathrm{cm}^{2}$ must be tested. Even after NIF and LMJ are completed, their shots will be too valuable to use for damage testing. The natural way to test a large optic is to scan it with a small, high repetition rate beam. Most available lasers for scan experiments have a Gaussian beamshape.

To relate results of tests with small Gaussian beams to expected results for flat top large beams, some assumptions must be made. For damage tests, we usually ignore any effect of conditioning. Some conditioning [1] can take place at high fluences $\mathrm{F}>10 \mathrm{~J} / \mathrm{cm}^{2}$ and this situation must be taken into account. It is assumed that the damage density at a point is a function of the maximum fluence observed at this point over the number of shots in the test. This assumption is reasonably adequate for fused silica, but questionable for KDP where conditioning is a strong effect [3].

A second typical assumption is the Weibull distribution (power law) for damage density $c(F) \sim F^{m}$. Here $c(F)$ is the density of damage sites produced by irradiation with fluence up to $F$. The Weibull index $\mathrm{m}$ for surface damage in fused silica is typically large, $\mathrm{m} \sim 5-10$. It should be noted that this assumption is not strictly necessary and has been adopted mainly because of scarcity of data. If damage tests are carried out at a number of test fluences with all conditions being held constant (particularly overlap spacing), then more general distributions can be derived from the measurements.

In raster scanning, the goal is to irradiate a large area with a small laser beam. The beam imprints form a scan lattice covering the plane. The way to relate this variable intensity lattice structure to flat top beam irradiation is the calculation of the effective beam area [2]. That is, the variable fluence exposure is equivalent to a flat top irradiation of the same peak fluence over a fraction of the total area. Calculations of effective area for different scan patterns in damage tests will be discussed in the first part of the paper.

Actual test beams exhibit intensity and pointing fluctuations. We consider the effects of fluence fluctuations and pointing accuracy on raster scan experiments in the second section.

*Correspondence: Email: rubenchik1@1lnl.gov, Telephone: 925-422-6131, Fax: 925-422-5099 
DKDP and KDP damage experiments clearly demonstrate a strong conditioning effect [3,4]. Preliminary irradiation of the sample by increasing the fluence of UV pulses results in a substantial increase of the subsequent damage threshold. Thus, the history of laser exposure is important. For experiments on conditioning, we assume that the important parameter is dose, i.e. the integral fluence at some point. Within an elementary cell of the raster lattice, the irradiation dose is due not only to the beam centered in the cell, but also to nearby neighbors. For experiments with large beam overlap, the input of many neighbors is important. To compare experiments made under different conditions, we will calculate the dose averaged over the elementary cell as a function of the overlap fraction $\mathrm{f}$. It is not clear that the dose is the sole important parameter for conditioning, but comparison of results of experiments with known doses can, at least, test this hypothesis.

It is desirable to have a numerical measure to compare different raster scan patterns. The ratio of minimum to peak fluence in a scan lattice cell can be used as a measure of variability [5]. This approach is simple and useful, but it takes into account neither the spatial distribution of intensity inside the cell nor the specific experimental setup. Damage experiments in fused silica are very sensitive to any local field maxima, and conditioning experiments are sensitive to the integrated dose We originally introduced the concept of "effective area" [2] to quantitatively measure the relative effectiveness of flat top and non-flat top beams in illuminating a sample in damage tests. This same definition is used in the present paper. The concept of effective area provides the means to compare different scan schemes and to select the most efficient one with respect to use of sample area. In the same way, the dose defined in the present work allows comparison of Gaussian beam scan conditioning experiments with ideal, flat top beam conditioning.

In the conclusion, we summarize our results, and discuss the limits of the assumptions made.

\section{THE LATTICE OF BEAM IMPRINTS AND EFFECTIVE AREA}

Small beam raster scanning produces an array of beam imprints covering the plane with a periodic lattice. Consider first the simple square lattice shown in Fig.1. The beam centers are spaced by distance 2a in both the vertical and horizontal directions. For the Gaussian beam

$$
F=F_{0} e^{-\frac{r^{2}}{b^{2}}}
$$

it is convenient to relate lattice spacing a to the overlap fraction $f$ and Gaussian beam radius $b$ via $a=b \sqrt{\ln (1 / f})$. For example, $\mathrm{f}=0.9$ means that the beams overlap at $90 \%$ of peak fluence $\mathrm{F}_{0}$. In this case, $a=0.325$ b. The Gaussian beam has maximum fluence $F_{0}$ only at the beam center.

To relate the damage produced by raster scanning to that produced by a flat top beam, we will assume that the density of defect $\mathrm{c}(\mathrm{F})$ has the Weibull distribution.

$$
c(F)=d F^{m}
$$

with $\mathrm{d}$ being a constant. Typically, the Weibull index is large, $\mathrm{m}>5$.

A flat top beam with fluence $F_{0}$ and area $S$ produces $c\left(F_{0}\right) S$ damage sites. The Gaussian beam produces

$$
\int c(F) d x d y \equiv c\left(F_{0}\right) S_{e f f}
$$

damage sites. Here $\mathrm{F}_{0}$ is the peak fluence and the integral is over a unit cell, of area $\mathrm{S}$, centered on a beam, so that this single beam provides the peak fluence for every point in the cell. Such cells are called WignerSeitz cells in condensed matter physics. The expression (1.3) defines the effective area $\mathrm{S}_{\text {eff }}$, i.e., the fraction of the area S effectively illuminated by the Gaussian beam. 


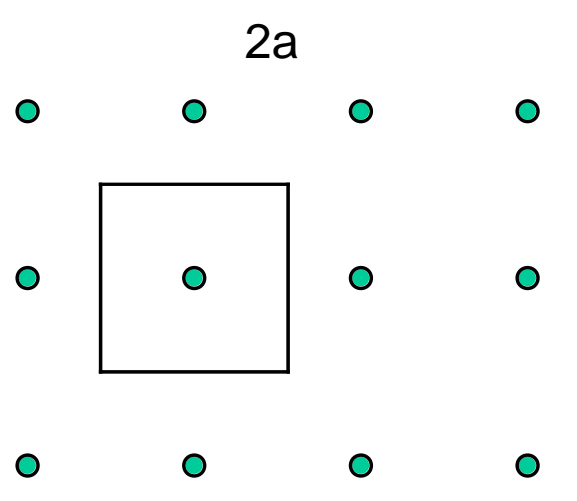

Fig.1: Square array of beam imprints. Dots signify beam centers. The distance between the centers is $2 \mathrm{a}$ in both the vertical and horizontal directions. Within each square unit (Wigner-Seitz) cell, every Fig.2: The spatial pattern of fluence produced by raster scan for a square lattice. One sees the square unit cells. point gets its maximum fluence from the beam at its center.

For an isolated Gaussian beam given by (1.1)

$$
S_{e f f}=\frac{\pi b^{2}}{m}
$$

The meaning of this result is that the area one would expect for a Gaussian, $\pi \mathrm{b}^{2}$, is reduced since the Gaussian 1/e radius of $F^{m}$ is reduced from the radius b of $F$ by a factor of $V_{m}$.

We can easily carry out the calculation of effective area given by Eq.(1.3) for the square lattice of Fig.(1). The calculated effective area for this case is given by [2].

$$
\frac{S_{\text {eff }}}{S}=\frac{\pi}{4}\left[\frac{\operatorname{Erf}(u)}{u}\right]^{2} ; \quad \text { with } u=\sqrt{m \ln (1 / f)}
$$

Here $\mathrm{S}=4 \mathrm{a}^{2}$ is the area of the elementary cell. The expression (1.4) indicates the fraction of the sample, which is effectively used in a raster scan experiment. The dependence of $S_{\text {eff }} / S$ on overlap $f$ for different Weibull indices $\mathrm{m}$ is shown in Fig.3. One sees that the curve is steeper for higher $\mathrm{m}$ so that small pointing (overlap) errors will result in larger variations of effective area, and thus affect damage density estimates, for larger $m$ at high overlap.

It is natural to ask if another scan pattern has a higher effective area and thus uses the scanned area more efficiently. Besides the square lattice, we may consider rectangular, parallelogram and hexagonal lattices as candidates. It is easy to check that the most efficient rectangular lattice is the square one.

Similarly, the parallelogram lattice of equal cell area shown in Fig. 4 can be shown to be analogous to the rectangle. That is, maximum use of the scanned area is achieved when the parallelogram has height $2 \mathrm{~h}$ equal to its base $2 \mathrm{a}$ when it has the same effective area as the square. The effective area is less for unequal height and base. 


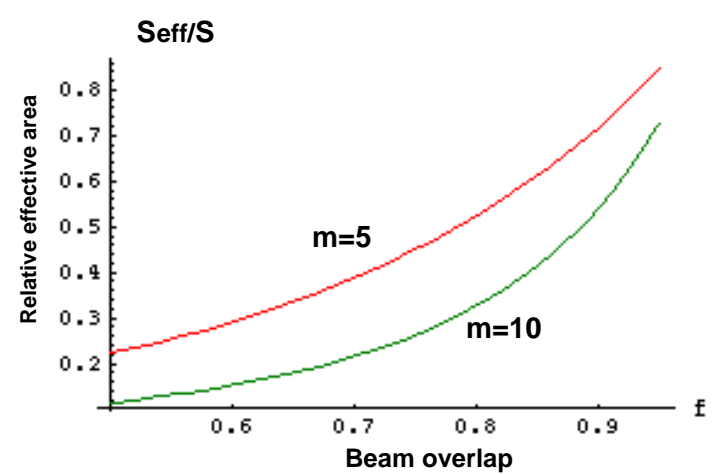

Fig.3: Relative effective area for square scan lattice as function of beam overlap $\mathrm{f}$ for Weibull indices 5 and 10 . The curve with larger $m$ is steeper.

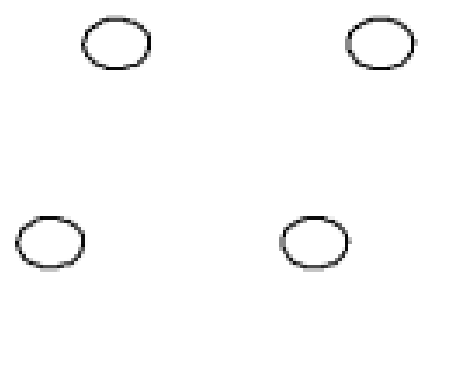

$\bigcirc$

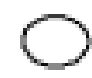

Fig.4: The array of beam imprints marking the position of the beam centers for a parallelogram lattice. The vertical distance between the rows is $2 \mathrm{a}$.

Consider now a hexagonal or triangular lattice (Fig.5). The distance between centers in every row is 2a as for the square lattice, but the distance between rows is smaller, $\sqrt{3} \mathrm{a}$. The centers of even rows are positioned between the centers in odd rows (see Fig.6). The elementary cell is a hexagon. To calculate the effective area, the integral in (1.3) must be taken over this hexagon. To calculate this integral, we divide the hexagon into its six equilateral triangles. The integral over the triangles is conveniently calculated in polar coordinates as follows.

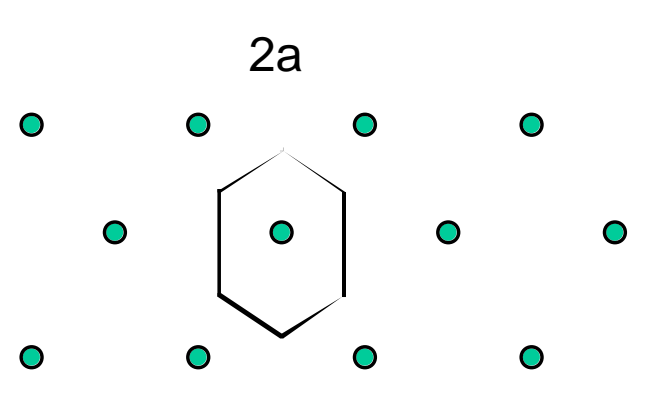

Fig.5: The hexagonal lattice. The Wigner-Seitz cell used to calculate the effective area is the hexagon, with width $2 \mathrm{a}$ and height $4 \mathrm{a} / \sqrt{ } 3$.

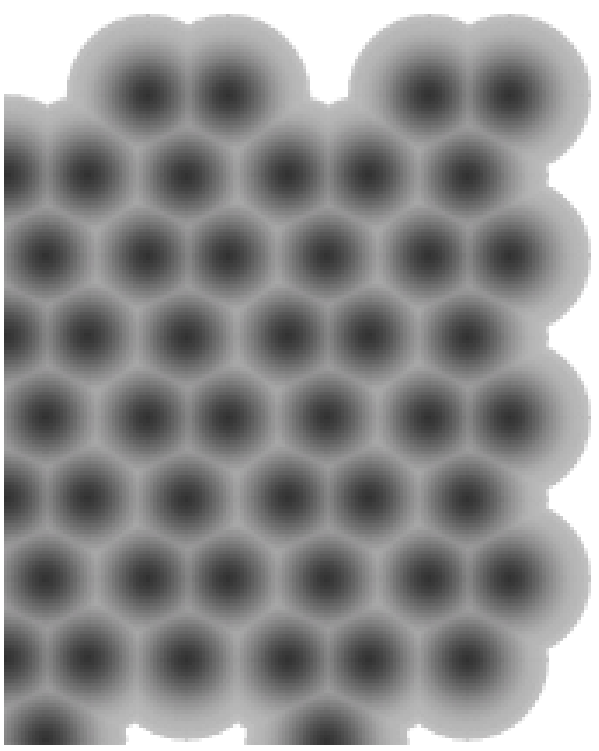

Fig.6: The spatial pattern of fluence produced by raster scan for the hexagonal lattice. The WignerSeitz structure is clearly seen.

Consider the representative triangle whose base is vertical. It's height is a and each side is $2 \mathrm{a} / \sqrt{3}$ in length. By symmetry, we need consider only the right triangle making up half the equilateral triangle. This triangle has area $\mathrm{S}=\mathrm{a}^{2} / 2 \sqrt{3}$. Note that for angle $\phi$, the maximum radius is $\mathrm{r}_{\max }(\phi)=\mathrm{a} / \cos (\phi)$. Then we have 
$\mathrm{S}_{\mathrm{eff}}=\int_{0}^{\pi / 6} \mathrm{~d} \phi \int_{0}^{\mathrm{r}_{\max }} \mathrm{dr} \mathrm{r} \exp \left(-\mathrm{mr}^{2} / \mathrm{b}^{2}\right)$

so

$\mathrm{S}_{\mathrm{eff}} / \mathrm{S}=\frac{\sqrt{ } 3}{\mathrm{u}^{2}} \int_{0}^{\pi / 6} \mathrm{~d} \phi\left(1-\exp \left[-\mathrm{u}^{2} / \cos ^{2}(\phi)\right]\right)$

where $u^{2}=m \ln (1 / f)$ as before.

The effective area is determined by the same parameter $u$ as in the case of the square lattice. The plot of $\mathrm{S}_{\mathrm{eff}} / \mathrm{S}$ versus $\mathrm{u}$ for different Weibull indices is shown in Fig.7.

One sees that the dependence on $u$ is different for square and hexagonal lattices and that the most efficient lattice depends on beam overlap $\mathrm{f}$. The relative difference between the effective areas for square and hexagonal lattices as a function of $\mathrm{u}$ is shown in Fig.8. More exactly we plot $\left(\mathrm{S}_{\mathrm{eff}}(\right.$ hex $\left.)-\mathrm{S}_{\mathrm{eff}}(\mathrm{square})\right) /$ $\mathrm{S}_{\text {eff }}$ (square) for equal spacing a. One can see that the hexagonal lattice is more efficient for most typical overlaps and Weibull indices. The relative difference can be large for small overlap, the hexagonal lattice can use $50 \%$ more sample surface than the square lattice does. One must also remember that the hexagonal lattice needs $2 / \sqrt{ } 3 \sim 1.15$ times as many shots to cover the same scan area if the lattice spacing a is the same for both.

Effective areas for the square and hexagonal lattices for specific values of $m$ and overlap are given in Table 2.

\begin{tabular}{|l|l|l|l|l|}
\hline & $\mathrm{m}=5, \mathrm{f}=0.9$ & $\mathrm{~m}=5, \mathrm{f}=0.5$ & $\mathrm{~m}=10, \mathrm{f}=0.9$ & $\mathrm{M}=10, \mathrm{f}=0.5$ \\
\hline $\mathrm{u}$ & 0.478 & 1.227 & 0.676 & 1.735 \\
\hline $\mathrm{S}_{\text {eff }} / \mathrm{S}$ (square) & 0.863 & 0.439 & 0.750 & 0.253 \\
\hline $\mathrm{S}_{\text {eff }} / \mathrm{S}$ (hex) & 0.78 & 0.49 & 0.71 & 0.32 \\
\hline
\end{tabular}

Table 2: Effective areas for different overlaps and Weibull indices for square and hexagonal lattices

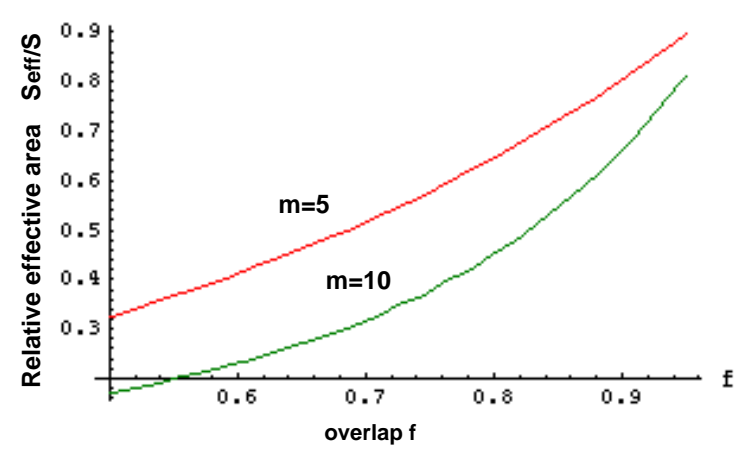

Fig.7: The effective area vs. overlap for hexagonal scan lattice. The green (lower) curve corresponds to $\mathrm{m}=10$, red (upper) to $\mathrm{m}=5$.

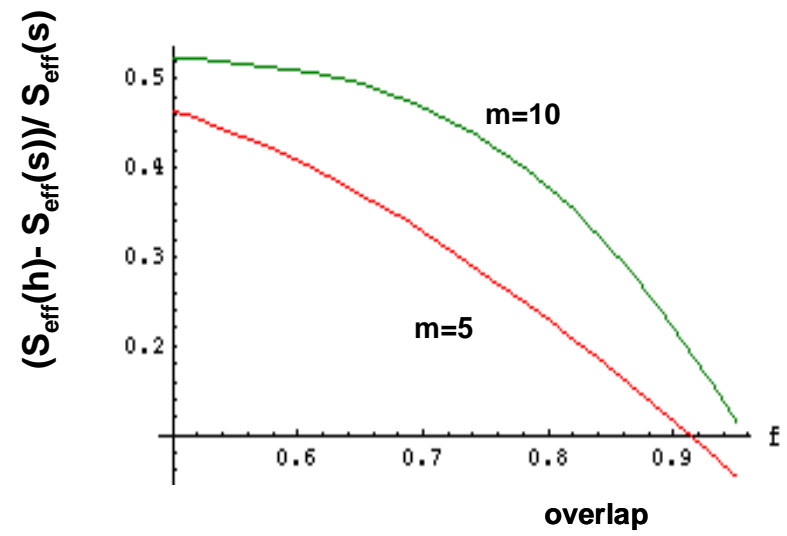

Fig. 8 The difference in effective areas between hexagonal and square lattices with the same overlaps. The upper curve corresponds to $m=10$, lower to $m=5$. 


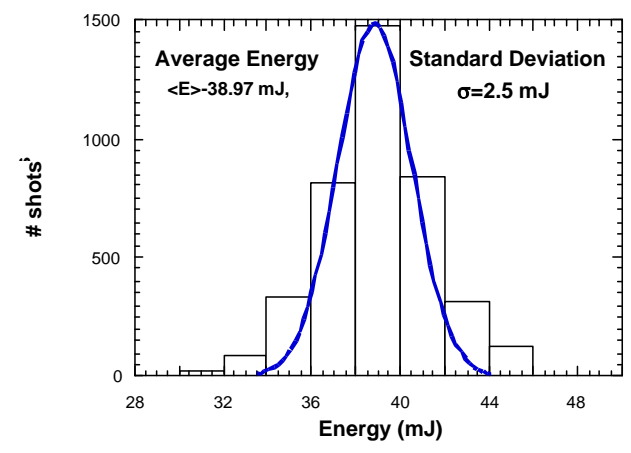

Fig.9: Measured energy fluctuations in the SOT beam. The mean energy is $38.97 \mathrm{~mJ}$, and the rms deviation is $\sigma=2.5 \mathrm{~mJ}$. The curve is a normal distribution for these values of mean energy and $\sigma$ (Courtesy of D.Hahn).

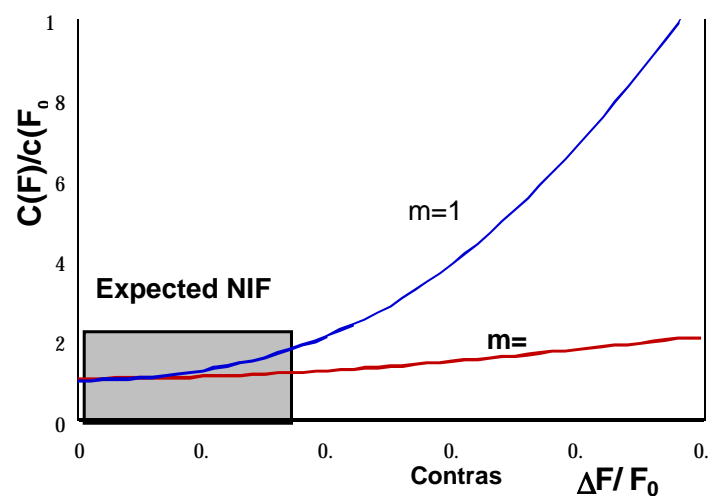

Fig.10: Increase in damage density $\mathrm{c}(\mathrm{F}) / \mathrm{c}\left(\mathrm{F}_{0}\right)$ as a function of rms energy fluctuations $\Delta \mathrm{F}$ for $\mathrm{m}=5$ and 10 .

\section{BEAM ENERGY FLUCTUATIONS.}

In our estimates above, we assumed that all shots have the same fluence and their positioning is perfect. Actual beam energy fluctuates and the pointing is not perfect. A histogram of the LLNL Small Optic Tester (SOT) laser energy distribution in a 4000 shots series is shown in Fig.9. These fluctuations produce uncertainty in the determination of damage density. If $\mathrm{P}(\mathrm{F})$ is the probability to have a shot with fluence $\mathrm{F}$, the observed damage density is given by the expression

$$
c(<F>)=\int P(F) c(F) d F
$$

Here $c(F) \sim F^{m}$ is the damage density introduced above. The damage density for the normal distribution (Gaussian) fit and actual distributions are given in Table 3 normalized by the density corresponding to the mean fluence.

\begin{tabular}{|l|l|l|l|}
\hline & Flat-top & Normal distribution & Actual distribution \\
\hline $\mathrm{m}=5$ & 1 & 1.021 & 1.155 \\
\hline $\mathrm{m}=10$ & 1 & 1.095 & 1.544 \\
\hline
\end{tabular}

Table 3: Relative damage density for flattop Gaussian and actual fluence distributions. Mean energy is $38.97 \mathrm{~mJ}, \sigma=2.5 \mathrm{~mJ}$.

We see that the energy fluctuations result in an overestimate of damage density compared to a constant fluence beam. For the normal probability distribution

$$
P(F)=\frac{1}{\Delta F \sqrt{\pi}} e^{-\frac{\left(F-F_{0}\right)^{2}}{\Delta F^{2}}}
$$


and Weibull defect density the integral (2.1) can be evaluated by the method of steepest descent [2] and the expected damage density are given by the expression:

$$
c(F)=c\left(F_{0}\right) \sqrt{\frac{4 b^{2}}{4 b^{2}+2 m}} \operatorname{Exp}\left[\frac{m^{2}}{4 b^{2}+2 m}\right]
$$

Here $\mathrm{c}\left(\mathrm{F}_{0}\right)$ is the damage density for a flat top beam with fluence $\mathrm{F}_{0}, \mathrm{~m}$ is the Weibull index, $\mathrm{b}=\mathrm{F}_{0} / \Delta \mathrm{F}$ is the inverse of the temporal "contrast", and $\Delta \mathrm{F}$ is the rms distribution width. Fluctuations are tolerable when they change $\mathrm{c}(\mathrm{F})$ less than 2 times. The increase in damage density as a function of $\Delta \mathrm{F} / \mathrm{F}_{0}$ is shown in Fig.10. One can see that for temporal contrast smaller than $30 \%$, the effect of fluctuations on damage density determination is minor. Let us note that this single shot result is very different from estimates of the effect on optics lifetime of spatial energy fluctuations [6]. The wandering of hot spots in the beam eventually produces a situation where every point is irradiated by the maximum possible fluence, and even a small beam contrast increase can greatly increase the amount of damage over many shots.

\section{BEAM POINTING FLUCTUATIONS}

Consider now fluctuations in beam pointing which lead to beam wander about the intended target at the center of the elementary cell. For simplicity we consider the square lattice only. We assume the mean displacement is small in comparison with the cell size a. For the square lattice, the effective area for a beam displaced by $\mathrm{x}$ and $\mathrm{y}$ from the cell center in the horizontal and vertical directions, respectively, is given by

$$
\frac{S_{e f f}}{S}=\frac{\pi}{16 u^{2}}\left(\operatorname{Erf}\left[u\left(1+\frac{x}{a}\right)\right]+\operatorname{Erf}\left[u\left(1-\frac{x}{a}\right)\right]\right)\left(\operatorname{Erf}\left[u\left(1+\frac{y}{a}\right)\right]+\operatorname{Erf}\left[u\left(1-\frac{y}{a}\right)\right]\right)
$$
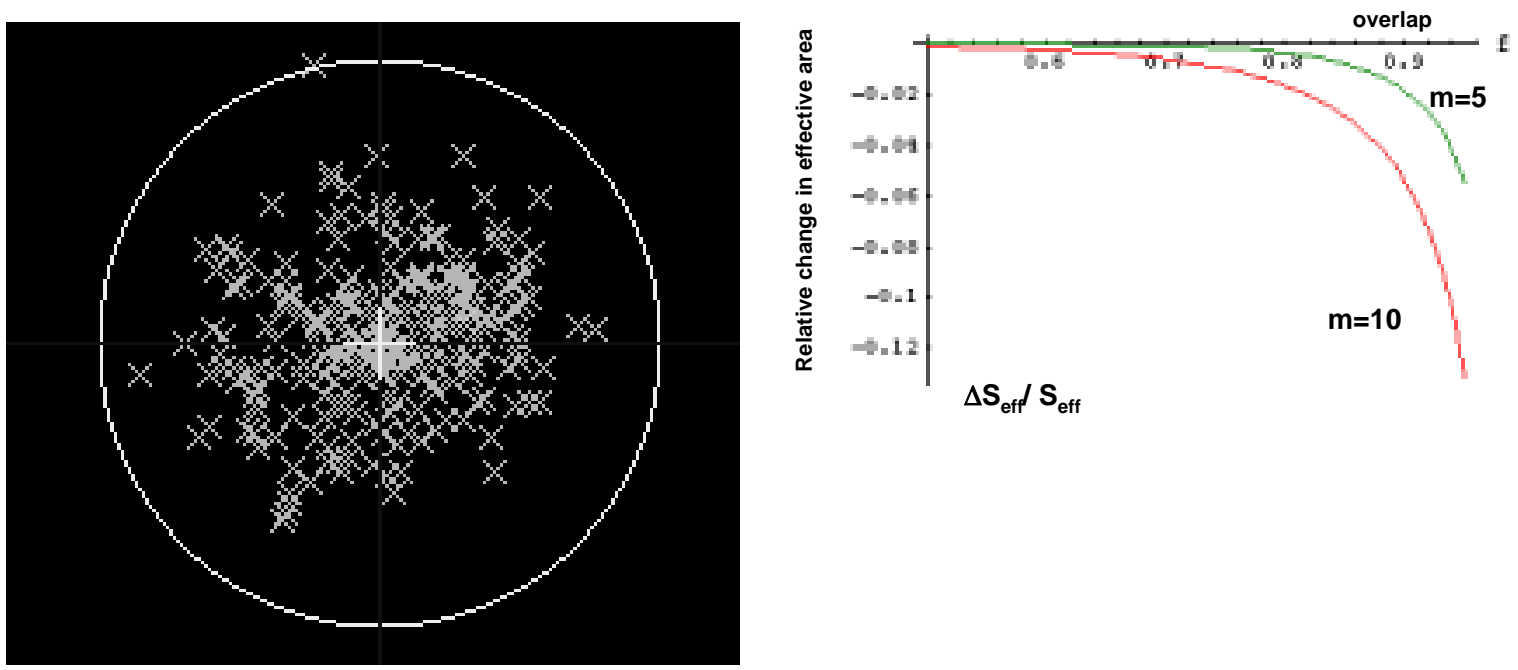

Fig.11 SOT beam wandering. Beam radius $b=300 \mu \mathrm{m}$, maximal deviation $\mathrm{r}=110 \mu \mathrm{m}$, rms deviation $\sigma=20 \mu \mathrm{m}$

Fig.12 Variation of effective area as a function of overlap for $\sigma / \mathrm{b}=0.2$

For small displacements, after expansion and averaging over the distribution of displacements we have 


$$
\frac{S_{e f f}}{S}=\frac{\pi}{4}\left(\frac{\operatorname{Erf}[u]}{u}\right)^{2}\left(1-\frac{2}{\sqrt{\pi}} \frac{u e^{-u^{2}}}{\operatorname{Erf}[u]} \frac{<x^{2}+y^{2}>}{a^{2}}\right)
$$

where $<\mathrm{x} 2+\mathrm{y} 2>$ indicates the mean square displacement. One sees that beam wandering always decreases the effective area, hence leads to an underestimate of the defect density if wander is not taken into account in a damage experiment. The effect of pointing fluctuations is determined by the mean square displacement. This value is independent of details of the distribution function and can be directly measured in experiments, e.g. with burn paper. For the SOT laser, the measured value $\sqrt{ }\left\langle r^{2}>\right.$ is about $20 \mu \mathrm{m}$ for beam radius $\sim 300 \mu \mathrm{m}$. As a result, the variation of effective area due to beam wandering is less then $1 \%$ according to equation (3.2).

The variation of effective area with overlap is shown in Fig. 12 for fixed level of beam wander $\sigma / b=0.2$. We conclude that beam wander is more important for large overlap. For small wander, i.e. $\sigma / b<<1$, the variation of effective area is small for typical experimental parameters.

\section{DKDP CONDITIONING AND EFFECTIVE DOSE}

Unlike fused silica, surface damage measurements in DKDP are complicated by the requirement for laser conditioning to prevent unacceptable levels of bulk damage at NIF fluences. Unfortunately, we do not have a reliable theoretical description of DKDP conditioning, or even a qualitative understanding of the process. We can assume, however, that conditioning is the result of annealing of some absorbing centers. In this case, it is natural to assume that the degree of conditioning will be determined, in part, by the total energy dose irradiating a given point. The dose accumulates the effects of all shots that hit a given point. In practice, conditioning fluences must be low enough not to damage the sample and high enough to have an effect. For example, there may be an activation energy. Below, we calculate the sensitivity of the dose to beam overlap f., disregarding any restrictions on fluence and the influence of shot history. For illustration, we consider the square lattice only. Various conditioning schemes are discussed in [5, 7].

The total relative dose $\mathrm{D}$, for a Gaussian beam with peak fluence $\mathrm{F}_{0}$ and radius $\mathrm{b}$ on a square lattice of spacing $2 \mathrm{a}$, relative to a flat-top beam of peak fluence $\mathrm{F}_{0}$, can be calculated as follow

$$
D=\frac{\int F(x, y) d x d y}{4 a^{2} F_{0}}=\int_{-a-a}^{a} \int_{s=-\infty}^{a} \sum_{p=-\infty}^{\infty} e^{-\frac{(x-2 s a)^{2}+(y-2 p a)^{2}}{b^{2}}} d x d y
$$

The sums over $\mathrm{s}$ and $\mathrm{p}$ include the contributions of neighboring cells. Integration is over the elementary cell. Physically, $D$ denotes the number of shots with a flat top beam with fluence $F_{0}$ needed to get the same dose. Integration over $\mathrm{x}$ and $\mathrm{y}$ can be completed independently and we find

$D=\frac{\pi}{16 u^{2}} \sum_{s=-\infty}^{\infty} \sum_{p=-\infty}^{\infty}(\operatorname{Erf}(u(1-2 s))+\operatorname{Erf}(u(1+2 s)))(\operatorname{Erf}(u(1-2 p))+\operatorname{Erf}(u(1+2 p)))$

where $u=\sqrt{\ln (1 / f)}$

The single term with $\mathrm{s}=\mathrm{p}=0$, the contribution of the central beam, is just the result (3.1) for $\mathrm{m}=1$. Due to the even symmetry in $s$ and $p$, we can write the summation over positive $s$ and $p$ values only:

$$
\sum_{s=-\infty}^{\infty}(\operatorname{Erf}(u(1-2 s))+\operatorname{Erf}(u(1+2 s)))=2\left(\operatorname { E r f } \left(u \left(+\sum_{s=1}^{\infty}(\operatorname{Erf}(u(2 s+1))-\operatorname{Erf}(u(2 s-1)))\right.\right.\right.
$$

Truncating the sum at some finite $\mathrm{N}$ we have 
$\operatorname{Erfu}+\sum_{s=1}^{N}(\operatorname{Erf}(u(2 s+1))-\operatorname{Erf}(u(2 s-1)))=\operatorname{Erf}(2 N+1) u$

For large $\mathrm{N}, \operatorname{Erf}(2 \mathrm{Nu}) \sim 1$ and for the dose we find

$$
D=\frac{\pi}{4 u^{2}}
$$

A plot of the relative dose D, as a function of beam overlap $f$ is given in Fig.13.

The result (4.3) allows estimation of the number of neighboring beams that contribute significantly for conditioning. Erf(x) is close to 1 at $\mathrm{x} \sim 1$ with accuracy $20 \%$. Hence, the number of important neighbors is $\mathrm{N} \sim 1 / 2 \mathrm{u}$. For overlap $\mathrm{f}=0.9, \mathrm{~N} \sim 2$ and the total number of participating neighbors is $\sim 13$. The total relative dose $\mathrm{D}=8.2$ in this case.

A planned experiment on DKDP conditioning with the excimer laser employs a highly elongated shape, with a near Gaussian profile in the transverse direction.[5]. The sample will be scanned by the laser beam with velocity $\mathrm{v}$ perpendicular to the long axis, providing a gradual increase in incident fluence desirable for conditioning. This situation is a near one-dimensional analog of the scheme discussed above. The distance between the beam centers is determined by the scan velocity $\mathrm{v}$ and repetition rate $\mathrm{p}$ :

$$
2 \mathrm{a}=\mathrm{v} / \mathrm{p} \text {. }
$$

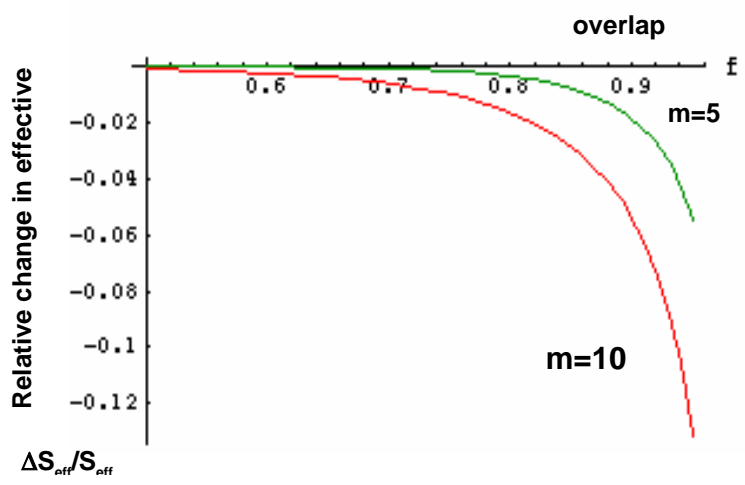

Fig.13: Relative dose as function of beam overlap fraction $\mathrm{f}$ for square lattice.

The calculation of the dose is similar to that presented above and gives

$$
D=\frac{\sqrt{\pi}}{2 u}
$$

where $\mathrm{a}$ in the expression for $\mathrm{u}$ is now determined by (4.4). We emphasize that, due to geometrical reasons, the dose in the one-dimensional case is less sensitive to overlap. 


\section{CONCLUSION}

Raster scan accelerated testing can be used to predict damage levels of large optics. Relating the results of scanning with a Gaussian beam to expected results for a flat top beam uses the effective area concept which indicates the equivalent flat top illuminated area. Different scanning schemes were considered. We demonstrated that for scans with fixed overlap and high Weibull index, the hexagonal lattice usually provides more efficient use of the sample (larger relative effective area) than the square lattice does. In our calculations, we assumed the Weibull model (power law) for the damage density as a function of fluence. This assumption is common for interpretation of our damage experiments, but extrapolation to fluence ranges not tested may not be reliable. The power law assumption is convenient, but not necessary. As long as the damage density distribution is of form $\mathrm{c}=\mathrm{g}(\mathrm{F} /<\mathrm{F}>)$, and several experiments are carried out under the same conditions (overlap, etc), then the shape and scale can be determined separately. Here $\mathrm{g}(\mathrm{x})$ is an arbitrary function.

We assume implicitly a uniform distribution of defects over the optic. This is not necessarily true, of course, but no strong evidence to the contrary has come to light.

We showed that test beam fluence fluctuations are not a large problem for raster scanning. If the rms fluence variation is less than $10 \%$ of the mean fluence, these fluctuations do not substantially affect measurements of damage density, even for high Weibull indices.

We demonstrated that pointing fluctuations reduce the effective area, and the apparent damage density if not taken into account. The effect is most pronounced for scans with high overlap. But, for pointing fluctuations small in comparison with the beam radius, beam wandering is not dangerous.

For conditioning experiments, the important process parameter is not the maximum fluence at some point, but, rather, the total dose of irradiation. To characterize conditioning, we introduced the effective dose, i.e. the dose averaged over an elementary cell. As a result, we can now compare experiments done with different scanning schemes and laser parameters. Specifically, it should be possible to check the hypothesis that conditioning is largely determined by the irradiation dose.

\section{ACKNOWLEDGEMENTS:}

This work was performed under the auspices of the U.S. Department of Energy by University of California, Lawrence Livermore National Laboratory under Contract W7405-Eng.48. We would like to thank A. Burnham, M. Runkel, M. Nostrand, D. Hahn and P. Whitman for helpful discussions.

\section{REFERENCES}

1. J.A. Menapace, B. Penetrante, D. Golini, A. Slomba, P.E. Miller, T. Parham, M. Nichols and J. Peterson, "Combined advanced finishing and UV laser conditioning for producing UV-damage-resistant fused silica optics", Proceedings XXXIII Annual Symposium on Optical Materials for High-Power Lasers 2001, SPIE 4679, 56-68 (2002).

2. M.D. Feit, A.M. Rubenchik, M.R. Kozlowski, F. Genin, L. Sheehan, S. Schwartz "Extrapolation of damage test data to predict performance of large-area NIF optics at $355 \mathrm{~nm}$ ", Proceedings XXX Annual Symposium on Optical Materials for High-Power Lasers 1998, Proc. SPIE 3578, 226-231, (1999).

3.R. Brusasco, B. Penetrante, J. Peterson, S. Maricle, J. Menapace," UV Laser conditioning for reduction of 351-nm damage initiation in fuse silica Proceedings XXXIII Annual Symposium on Optical Materials for High-Power Lasers 2001 SPIE 4679, 48-55 (2002).

4. M. Runkel, J. DeYoreo, W. Sell, D. Milam, "Laser conditioning study of KDP on the Optical Sciences Laser using large area beams," Proceedings XXIX Annual Symposium on Optical Materials for High-Power Lasers 1997, SPIE 3244, 51-63, (1998). 
5. M. Runkel, M. Nostrand, "Overview of raster scanning for ICF-class laser optics", Proceedings XXXIV Annual Symposium on Optical Materials for High-Power Lasers 2002 this proceedings.

6. M.D. Feit and A.M. Rubenchik, "Effect on estimated silica lifetimes of operating with average fluence near damage growth threshold and continued need for growth mitigation", LLNL memo NIF-0070225,

(Aug. 27, 2001).; M.D. Feit, "Expected new damage initiation sites due to random beam contrast on LAT

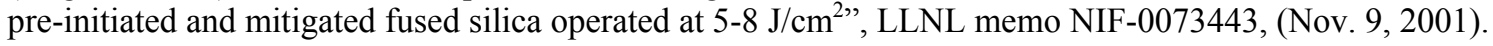
7. C.J. Stolz, L.M. Sheehan, S.M. Maricle, S. Schwartz, "A study of laser conditioning methods of hafnia silica multilayer mirrors" Proceedings XXX Annual Symposium on Optical Materials for High-Power Lasers 1998 SPIE 3578, 144-152, (1998). 